\title{
L'agglomération antique de Locmariaquer (Morbihan) État des connaissances
}

Lionel Pirault

\section{(2) OpenEdition}

\section{Journals}

Édition électronique

URL : http://journals.openedition.org/abpo/1462

DOI : $10.4000 / a b p o .1462$

ISBN : 978-2-7535-1489-8

ISSN : 2108-6443

Éditeur

Presses universitaires de Rennes

Édition imprimée

Date de publication : 20 mars 2003

Pagination : 7-27

ISBN : 978-2-86847-811-5

ISSN : 0399-0826

\section{Référence électronique}

Lionel Pirault, "L'agglomération antique de Locmariaquer (Morbihan) État des connaissances »,

Annales de Bretagne et des Pays de l'Ouest [En ligne], 110-1 | 2003, mis en ligne le 20 mars 2005,

consulté le 30 avril 2019. URL : http://journals.openedition.org/abpo/1462 ; DOI : 10.4000/abpo.1462

(C) Presses universitaires de Rennes 


\title{
L'agglomération antique de Locmariaquer (Morbihan) État des connaissances
}

\author{
Lionel PIRAULT \\ Archéologue à l'Institut National de Recherches Archéologiques Préventives
}

Le site antique de Locmariaquer est connu depuis le début du XVII ${ }^{\mathrm{e}}$ siècle, époque à laquelle Dubuisson-Aubenay dans son Itinéraire de Bretagne dépeint pour la première fois les vestiges de l'édifice gallo-romain du Er Castel au sud du bourg ${ }^{1}$. La description qu'il en fait en 1636 diffère peu de celle faite par M. de Robien un siècle plus tard en $1727^{2}$. Toutefois, le jugement qu'il porte sur ces " ruines considérables " diverge totalement de celui de Christophe-Paul de Robien puisqu'il affirme : "En ces restes de murailles il n'y a pas un seul brin d'ouvrage romain. " Il faut attendre près d'un siècle pour que Christophe-Paul de Robien, seigneur de Locmariaquer, "découvre tous les indices d'une grande et ancienne ville ${ }^{3}$ ".

C'est en effet à ce savant érudit que nous devons la première étude réalisée sur les antiquités gallo-romaines de Locmariaquer. Ces travaux ne manquent pas d'intérêt, notamment sa cartographie des vestiges archéologiques du bourg qui permet, aujourd'hui encore, de localiser les principaux édifices gallo-romains qui constituent une partie de la parure monumentale de cette agglomération antique (fig. 2). Nous ne connaissons pas la véritable cause de ces relevés. Sont-ils le fait d'une restructuration du bourg au XVIII ${ }^{\mathrm{e}}$ siècle, des travaux qui auraient permis de mettre au jour les restes de vestiges ou s'agit-il de ruines en élévation conservées dans le paysage? Dans cette dernière hypothèse on peut alors s'interroger sur l'absence d'indication concernant le théâtre au nord du bourg dont certains pans de

1. Dubuisson-Aubenay ne cite pas l'édifice gallo-romain de " Er Castel ", mais il le désigne sous le nom de " château de Julius Caesar ", Dubuisson-AubENAY, François-Nicolas Baudot, seigneur du Buisson, Itinéraire de Bretagne en 1636, avec notes et éclaircissements de Léon Maître et Paul Berthou, Nantes, édition de la Société des bibliophiles bretons et de l'histoire de Bretagne, t. IX et X, 1898-1902, p. 294.

2. RoBIEn, Christophe-Paul, Description historique, topographique et naturelle de l'ancienne Armorique, édité par Jean-Yves Veillard, Floch, Mayenne, 1974.

3. Closmadeuc, Gustave Thomas de, "Le Président de Robien archéologue ", Bulletin de la Société Polymathique du Morbihan, 1882, p. 51-56. 
murs sont encore en élévation au début du siècle dernier. Il peut également s'agir d'une initiative inspirée par l'instruction ministérielle adressée à l'Intendant de Bretagne vers le milieu du XvIII ${ }^{\mathrm{e}}$ siècle afin de " prendre soin des monuments nationaux surtout ceux qui se rattachent à l'époque celtique $^{4} "$.

\section{Figure 1 - Plan de situation géographique}

La position privilégiée de Locmariaquer permettait de contrôler l'entrée du Golfe du Morbihan ainsi que l'accès au chef lieu de cité Darioritum (Vannes)

(Plan et dessin : L. Pirault, 2002)

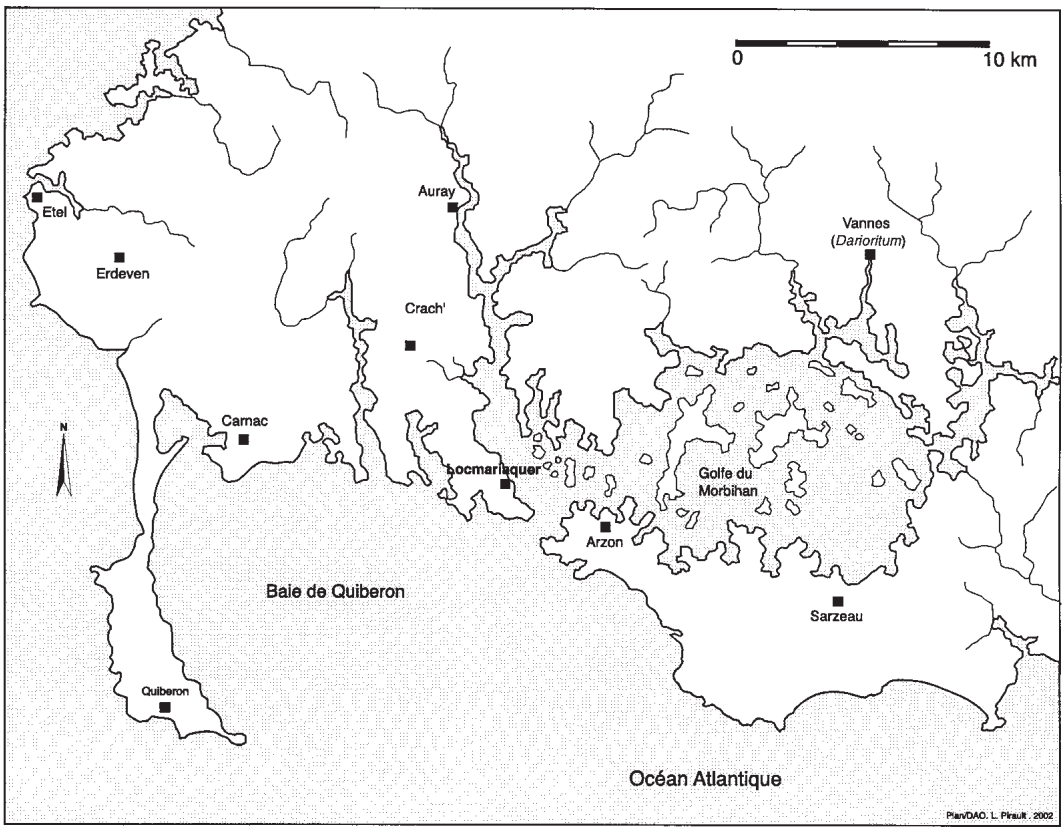

Il faut attendre le XIX ${ }^{\mathrm{e}}$ siècle et les travaux de M. Gaillard pour commencer à prendre conscience de l'importance de l'occupation antique. En 1834, se basant sur les travaux de son prédécesseur Christophe-Paul de Robien, et à partir de ses propres observations de terrain, il tente de déterminer la superficie du site gallo-romain de Locmariaquer. Selon cet auteur, le gisement antique implanté le long du littoral de la rivière d'Auray, sur une légère proéminence topographique, devait avoisiner les 13 hectares de superficie " de 600 mètres de longueur sur une largeur moyenne de 220 mètres ${ }^{5}$ ». En 1837, c'est au tour de Prosper Mérimée de décrire des vesti-

4. Société des Bibliophiles Bretons, Mélanges historiques, littéraires bibliographiques, monuments historiques, t. II, 1883, p. 255-258.

5. GAILlARD M., Recherches archéologiques sur Locmariaker, Annuaire statistique, Historique, administratif du Morbihan, Vannes, 1834, p. 40-63. 
Figure 2 - Plan et légendes des vestiges antiques relevés parde M. de Robien en 1727 dans le bourg de Locmariaquer (d'après comte de Caylus, 1762-1767, Recueil d'antiquités égyptiennes, étrusques, grecques, romaines et gauloises)

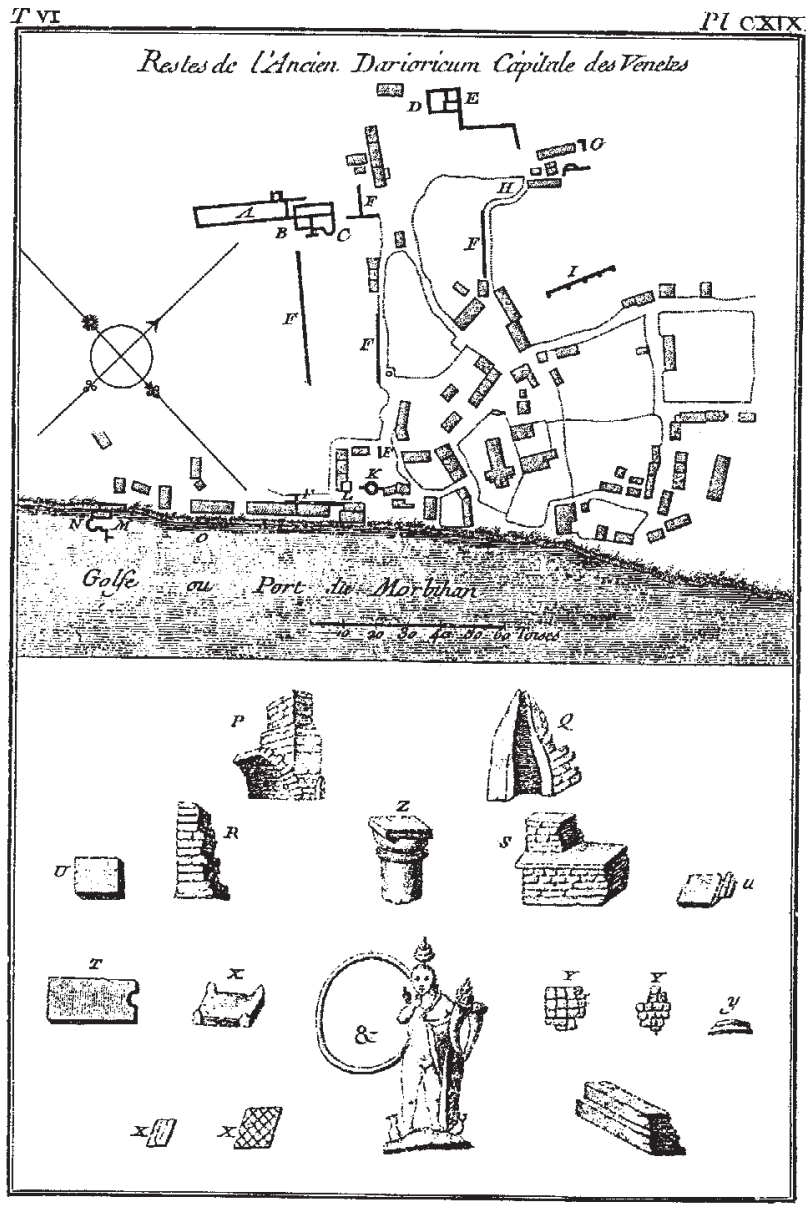

"Voici les renvois tels que M. de Robien les a marqués dans fon Manufcrit.

"A. Principaux reftes des anciens bâtimens. B. Partie qui a été voûtés \& dont l'élévation eft marquée par la lettre $P$. On a trouvé dans ces ruines les briques cotées $V$. C. Efpèce de cul-de-four large de feize pieds par le bas, \& d'un pied par le haut : on en voit l'élévation à la lettre $Q$. D. Autre bâtiment. E. Sallon pavé des grandes briques marquées $V$. fur un fond de ciment d'une dureté extrême. F. Anciens murs répandus de différens côtés bâtis en chaux \& en ciment. G. Coin de mur d'environ dix pieds hors de terre, dont l'élévation est marquée $R$. H. Lieu où l'on a trouvé des morceaux de marbre. I. Anciens murs avec des jambes de force. K. Tour d'environ douze pieds de diamètre, avec un mur plus épais que les autres. L. Sallon pavé de briques, où l'on a trouvé les mofaïques cotées $X \& Y$; l'une eft formée de petites pierres blanches; l'autre est femée de quelques pierres noires. M. Fondemens trouvés dans les grèves que la mer couvre de haute marée. N. Tour plus avancée dans la mer, \& qui a environ dix ou douze toifes : il y a des habitans qui ont vû d'anciens mur à plus de foixante toifes au delà. O. Lieu où l'on a trouvé le chapiteau de colonne marquée $Z$. P. Elévation de la partie voûtée marquée $B$ fur le plan. Q. Elévation de la partie voûtée en cul-defour, indiquée fur le plan $C$. R. Elévation du mur $G$. S. Mur conftruit de petites pierres entre des couches fort épaiffes de ciment, avec un cordon de grandes briques. T. Brique large de dix-huit pouces, fervant à faire le cordon des murs. V. Brique d'un pied en quarré \& d'un demi-pied d'épaiffeur, dont étoient pavés les fallons $E \& L$. u. Morceaux de briques trouvés dans les bâtimens $A \& B$. X. Morceaux de briques trouvés dans le fallon $L$. Y. Morceaux de mofaïque. $\boldsymbol{y}$. Fragment d'une urne trouvée dans le même fallon $L$. M. le Président de Robien dit ailleurs que ce vafe eft d'une terre brune \& très fine, de la nature de celles que les Etrufques ont travaillées. Z. Chapiteau de colonne trouvé dans la mer à l'endroi marqué $O$ sur le plan. \&. Petite Idole d'or d'environ deux pouces de hauteur \& du poids de deux louis; cette petite figure Panthée à été trouvée par un Pêcheur, à la pointe Nord-eft de Locmariaker. " 
ges antiques et particulièrement ceux de " Er Hastel " situés le long de la route de Kerpenhir au sud du bourg. Ses observations permettent déjà de mesurer l'importance des vestiges gallo-romains observables dans ce secteur du bourg :

"Lorsqu'en sortant du bourg de Locmariaker, on se dirige vers la métairie de Kerperai, on passe devant quelques jardins attenant aux dernières maisons; un mur ruiné, qui traverse ces jardins, attire d'abord l'attention par la régularité de son appareil. En l'examinant, on y reconnaît les assises parallèles, le parement de petites pierres bien taillées qui caractérisent les constructions romaines. Ce mur se prolonge assez loin et touche à d'autres substructions semblables. La partie inférieure de la façade d'une maison de pauvre apparence présente le même appareil; enfin, à quelque distance de là, on a découvert dernièrement une immense quantité de tuiles à crochets et un épais massif de pierre et de ciment ${ }^{6} \ldots$..

Quelques années plus tard en 1847, Cayot-Delandre remarquera que certaines maisons du bourg semblent être construites sur des fondations de bâtiments gallo-romains :

"Toutes les clôtures des champs qui avoisinent le bourg sont composées de petites pierres uniformément taillées qui proviennent certainement de la démolition de murailles romaines, et plusieurs maisons du bourg ont des parties qui présentent le même appareil. Les débris de tuiles et de poteries romaines y rougissent le sol $^{7} \ldots$ "..

C'est peut-être Ogée qui dans son Dictionnaire Historique et géographique de la province de Bretagne publié en $1843^{8}$, apporte le plus de précision sur l'étendue et l'importance du site qu'il pensait être l'emplacement de l'ancienne cité de Darioritum. Il émet également pour la première fois, à partir d'éléments recueillis sur place, l'hypothèse de l'existence d'une sorte " d'enceinte " dont les traces seraient encore perceptibles (à l'époque) au sudest du bourg et en bordure de littoral :

" [...] Ainsi, revenant au carré de maçonnerie dont M. Gaillard a parlé, et où la tradition place l'emplacement d'un château gothique bâti sur les ruines d'une ancienne forteresse, on remarque d'abord, dans la partie la plus élevée de ce carré, deux murs construits parallèlement, et se dirigeant vers le midi : en suivant le prolongement du second de ces murs, on retrouve dans les clôtures de deux petits courtils des indices de la suite de ce mur; mais de l'autre côté du chemin, après avoir traversé une aire à battre, le mur se présente dans une assez grande largeur hors de terre, bien conservé, et suivant toujours la même direction. À l'extrémité du pâtis auquel il sert de clôture, il semble s'arrêter; le placement des pierres indique un angle, et cependant on le

6. Merimee, Paul, Notes d'un voyage dans l'Ouest de la France, Bruxelles, 1837, p. 260-262.

7. Cayot-Delandre, Le Morbihan, son histoire et ses monuments, Rennes/Vannes, A. Candézan, 1847, $561 \mathrm{p}$.

8. OgeE, Jean-Baptiste, Dictionnaire historique et géographique de la province de Bretagne, Nantes, édition 1843, 2 vol. L'ouvrage de Jean-Baptiste Ogée a été publié en quatre volumes entre 1778 et 1780 à Nantes. L'édition originale a fait l'objet de rééditions en 1824,1843 et 1853 qui ont été augmentées et modifiées par différents auteurs tels que A. Marteville, P. Varin, Ducrest de Villeneuve, M. de Blois, etc. 
voit se prolonger plus loin. Ce pan de mur est une preuve que la ville était cernée. Il paraît bien démontré que l'enceinte se prolongeait jusqu'à la hauteur de cette dernière muraille. Une maison construite au-dessous, sur les bords du quai, a sa fondation assise sur d'anciennes maçonneries $[\ldots]^{9}$."

Ogée est aussi le premier auteur, après l'abbé Mahé en $1825^{10}$, à rapporter l'existence " d'un cirque " dont certains murs étaient encore visibles en 1820 :

"On reconnaît dans le Nordet la courbe bien apparente d'un cirque. Les spectateurs placés sur les gradins pouvaient apercevoir le Morbihan, dont les rivages et les nombreuses îles, alors couvertes de bois, offraient une admirable décoration pour le fond de la scène. Des portions des murs du cirque existaient encore en $1820^{11}$."

Malheureusement pour le site antique, les savants du XIX ${ }^{\mathrm{e}}$ siècle, furent nettement plus inspirés par le courant de pensée celtomane et préférèrent disserter sur les "monuments celtiques " de la commune. Pendant près d'un siècle et demi, l'intérêt pour le site gallo-romain s'atténuera considérablement pour ne susciter une nouvelle attention que bien des années plus tard. Mises à part quelques rares exceptions, on peut dire que pendant toute la durée du XIX ${ }^{\mathrm{e}}$ siècle et une bonne partie du $\mathrm{XX}^{\mathrm{e}}$ siècle, la plus grande majorité des relations régulières de découvertes ou de fouilles à Locmariaquer sera essentiellement consacrée aux monuments mégalithiques et à l'étude de leur mobilier.

Les premières et véritables réflexions sur le site antique de Locmariaquer sont assez irrégulières et très imprécises. Si l'on excepte les quelques sondages réalisés à la fin des années 1980 sur le site du Lehuie (Er Hastel) ${ }^{12}$ et l'opération de sauvetage urgent effectuée plus récemment à l'emplacement de l'école du Votten ${ }^{13}$, il n'existe que deux comptes-rendus ayant trait à des "fouilles " gallo-romaines à Locmariaquer. Le premier, réalisé en 1853 par le docteur Fouquet de Vannes est un document manuscrit très détaillé qui est conservé aux Archives départementales d'Ille-etVilaine $^{14}$. Ce dernier concerne la fouille d'une partie d'un édifice galloromain (vraisemblablement des thermes) dans le centre du bourg (la maison Le Rol). Ce document inédit est accompagné d'un plan de masse

9. OGEE, Jean-Baptiste, Dictionnaire..., op. cit., p. 519.

10. MAHE J. abbé, Essai sur les antiquités du département du Morbihan, Vannes, Galles Aîné, 1825, 500 p., 5 pl.

11. OgeE, Jean-Baptiste, Dictionnaire ..., op. cit., p. 519.

12. BARDEL, Jean-Pierre, Rapport de sondages. Lotissement de "Le Lehuie "à Locmariaquer, Service régional de l'Archéologie de la région Bretagne, DRAC, Rennes, 1987.

13. Pirault, Lionel, L'École du Votten (commune de Locmariaquer). Document final de synthèse. de fouille de sauvetage, Service régional de l'Archéologie de Rennes, DRAC Bretagne, 1996.

14. FOUQUET, $\mathrm{D}^{\mathrm{r}}$, Des monuments celtiques et des ruines romaines dans le Morbihan, extrait d'un rapport présenté à la Société polymathique du Morbihan sur la découverte d'une habitation gallo-romaine au milieu du bourg de Locmariaquer, Vannes, 1853, 8 p., 1 pl., Arch. dép. d'Ille-et-Vilaine, T 184. 
des vestiges qui ne permet malheureusement pas d'en localiser avec précision les emplacements. On peut cependant dire qu'ils se trouvaient dans des jardins situés au cœur d'un îlot proche de l'église paroissiale. Le dégagement partiel du théâtre au nord du centre bourg à l'emplacement de l'ancien cimetière par Gustave Thomas de Closmadeuc en $1893^{15}$, constitue la seconde relation qui demeure un document d'une grande précision topographique et architecturale.

\section{L'abside du chevet de l'église de Locmariaquer}

Elle présente un appareillage constitué par des arases régulières de petits moellons en granit (opus uitattum). Il n'est pas impossible que cette partie de l'église utilise le reliquat d'un édifice antérieur (peut-être de l'Antiquité tardive) (cliché, L. Pirault, 2002)

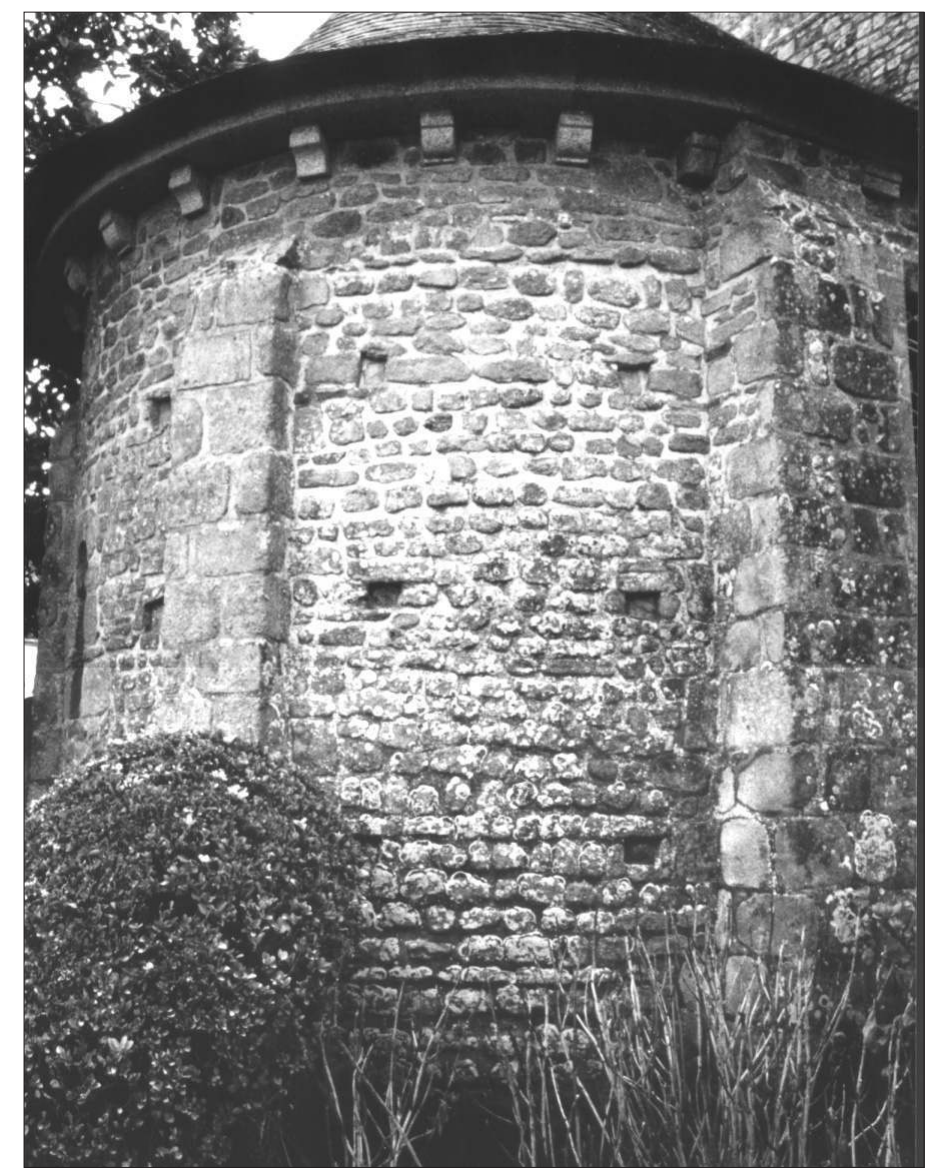

15. Closmadeuc, Gustave Thomas de, "Théâtre romain de Locmariaquer ", Bulletin de la Société polymathique du Morbihan, 1893, p. 181-192. 


\section{Figure 3 - Plan général de la commune}

L'axe de circulation antique qui desservait l'agglomération gallo-romaine s'est partiellement fossilisé dans le paysage sous la forme de limites parcellaires et de chemins vicinaux. On remarque également que les monuments mégalithiques les plus importants sont situés dans le périmètre proche de l'agglomération antique. Certains d'entre eux, tel que le Mané Rutual et l'ensemble monumental Grand Menhir, Table des Marchand y sont quasiment intégrés

(plan et dessin : L. Pirault, 2002)

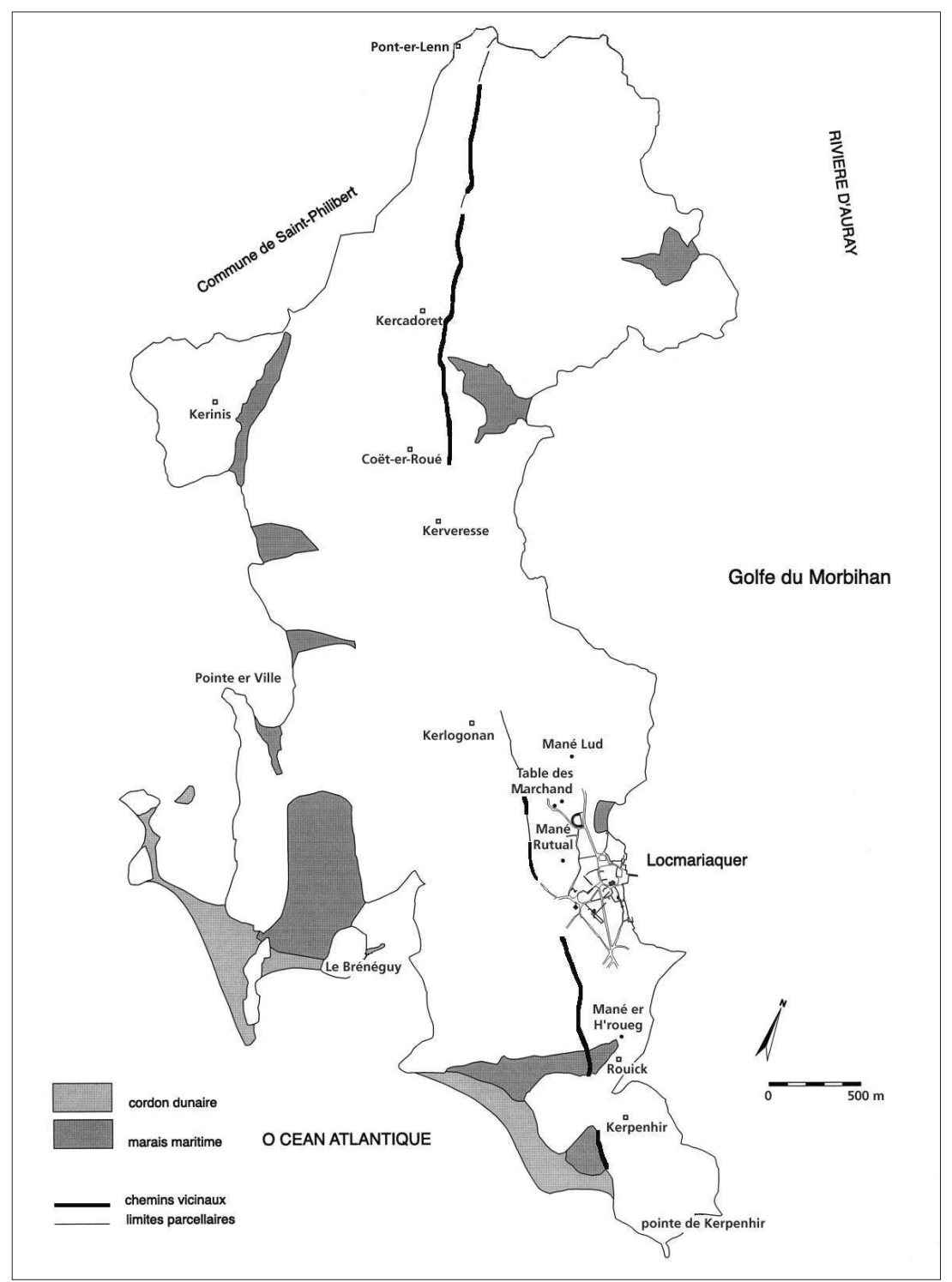




\section{Éléments de topographie}

La configuration topographique contemporaine de Locmariaquer s'insère dans un environnement maritime et terrestre dont la restitution précise pour l'Antiquité ne peut guère s'appuyer sur les composantes actuelles du paysage. Les changements environnementaux continus (facteurs naturels et anthropiques) ont profondément modifié la géographie des lieux et la présence de vestiges sur l'actuel estran implique que ce dernier était nettement plus en retrait à l'époque gallo-romaine.

Figure 4 - Plan de l'agglomération antique (plan et dessin : L. Pirault, 2002)

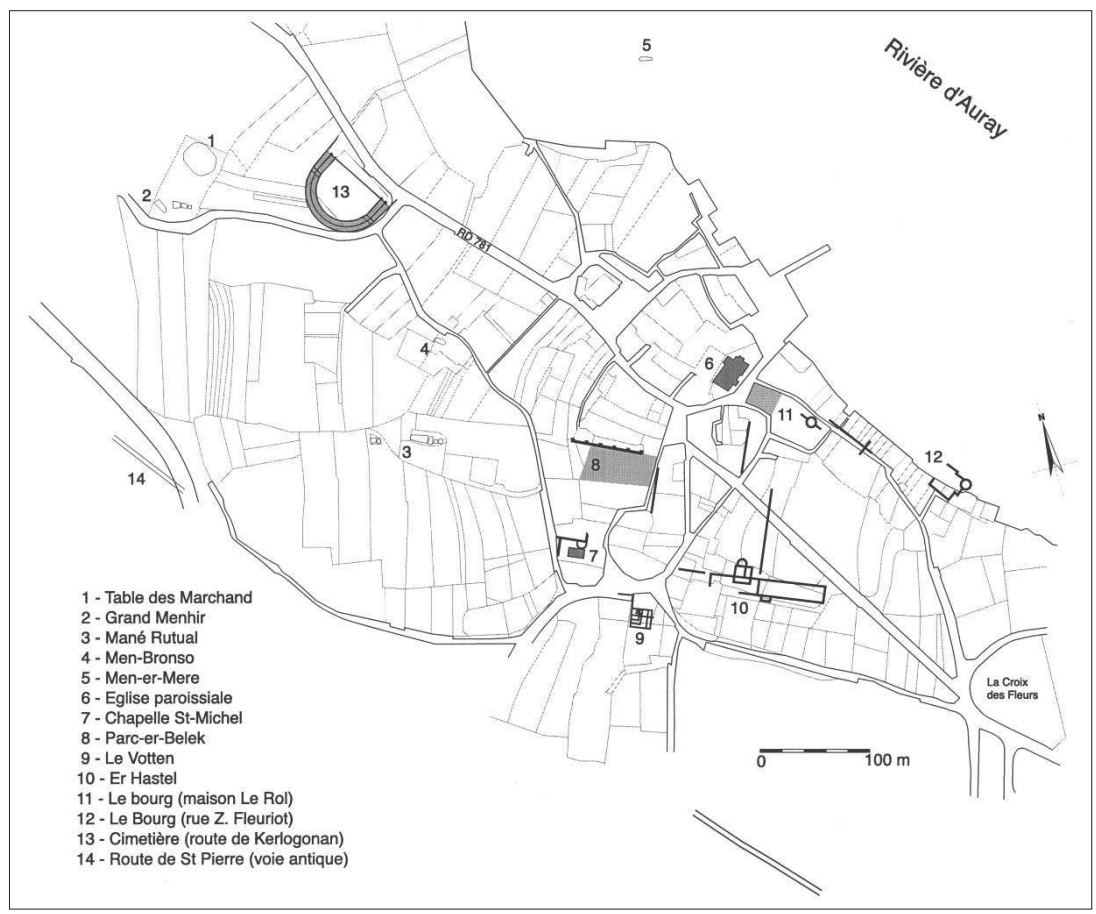

L'agglomération était reliée à Vannes (Darioritum) par une voie dont le tracé reste encore assez imprécis. Sur le territoire de Locmariaquer certaines formes parcellaires conservent l'empreinte d'un axe de circulation rectiligne qui scinde le paysage bocager au nord de la commune (fig. 3). Le tracé de cette voie est encore très perceptible entre le village de Ponter-Lenn et celui de Coët-er-Roué. Entre ces deux points, un chemin vicinal emprunte l'ancien cheminement de la voie jusqu'à la route départementale 781. On perd totalement sa trace entre le village de Kerveresse (au nord du hameau) et celui de Kerlogonan. À ce niveau, son tracé est tangible sous la forme de limites parcellaires continues jusqu'au bourg de Locmariaquer. Au-delà du bourg (vers le sud), la voie est encore relative- 
ment bien visible jusqu'à la pointe de Kerpenhir située à l'extrémité méridionale de la commune.

Le site antique de Locmariaquer s'étend sur une surface d'environ 20 hectares. Il est implanté à flanc de coteau le long du littoral et occupe une faible proéminence topographique d'une déclivité peu importante dont le point culminant (la chapelle Saint-Michel) se situe à 14 mètres NGF. Il est bien évident que ces limites restent tout à fait hypothétiques et nous ne les présumons que par la présence de certains édifices connus. Il englobe le centre bourg historique (église, enclos paroissial) et s'étire au sud, jusqu'au croisement de "La Croix des Fleurs" (route de Kerpenhir). Le Votten et le cimetière communal constituent les limites connues à l'ouest et au nord de l'agglomération antique (fig. 4).

\section{Le théâtre}

La plus grande partie des monuments connus est groupée dans la partie sud du bourg. Seul, le théâtre est implanté au nord à flanc de coteau à proximité des monuments du Nélud (Er Grah, Table des Marchand, Grand Menhir) ${ }^{16}$. Cet édifice a fait l'objet d'un dégagement partiel en 1893 par Gustave Thomas de Closmadeuc. La fouille débuta le 8 septembre 1893 et s'acheva le 14 octobre de la même année ${ }^{17}$. Elle permit de mettre au jour un vaste hémicycle de 160 mètres de pourtour composant une cavea formée par trois murs concentriques (fig. 5). La façade était constituée par un mur d'une longueur de 80 mètres d'une extrémité à l'autre de cet hémicycle.

" [...] Le monument découvert est situé sur le bord et à droite de la route qui mène d'Auray à Locmariaquer; à très peu de distance au nord des premières maisons du bourg [...]. Il est à 100 mètres seulement au sud-est du dolmen des Marchand; et à un peu plus de 100 mètres, à l'ouest du rivage de la baie de Locmariaquer. Le nom de Vinglé (carrière de pierre) lui est commun avec celui des pièces de terre voisines [...]. En certains endroits, la hauteur des murs dépasse un mètre. La présence dans les décombres d'une très grande quantité de briques larges et plates, si ce ne sont pas des briques de pavage, semblerait indiquer qu'elles formaient des assises dans la maçonnerie qui s'élevait au-dessus du sol, comme nous en avons des exemples à Locmariaquer même, dans le mur romain qui borde le chemin de Parc-Belek. Aucun dallage n'est en place, sauf dans l'espace compris entre les deux murets de refend. Là, le pavage était constitué par des blocs de granit. L'hémicycle que nous avons sous les yeux, avec ses trois murs courbes, concentriques,

16. En 1825, l'abbé J. Mahé dans son Essai sur les antiquités du département du Morbihan fait allusion à ce monument dont la configuration était encore perceptible dans le paysage " un cirque dont les murs ont été démolis de notre temps, mais dont on distingue encore la forme ", MAHE J. abbé, Essai sur les antiquités..., op. cit.

17. La Société polymathique du Morbihan a subventionné cette opération en allouant à Gustave Thomas de Closmadeuc et à J. Mahé, une somme de 300 francs auxquels le ministre de l'Instruction publique et des Beaux-Arts ajouta 300 francs pour l'exécution et la publication des travaux, Closmadeuc, Gustave Thomas de, "Théâtre romain de Locmariaquer ", Bulletin de la Société polymathique du Morbihan, 1893, p. 181-192. 
servait probablement de support à des gradins en bois; car nulle part dans les matériaux écroulés nous n'avons trouvé trace de voûtes, de marches, ni de sièges en pierre : au contraire beaucoup de cendres et de charbons, sur lesquels les fibres ligneuses se reconnaissent et de nombreuses chevilles et de nombreux clous de fer oxydés $[\ldots]^{18}$."

\section{Figure 5 - Plan du théâtre de Locmariaquer}

(par G. de Closmadeuc en 1893, Théâtre romain de Locmariaquer,

Bulletin de la Société polymathique du Morbihan, $p$. 185)

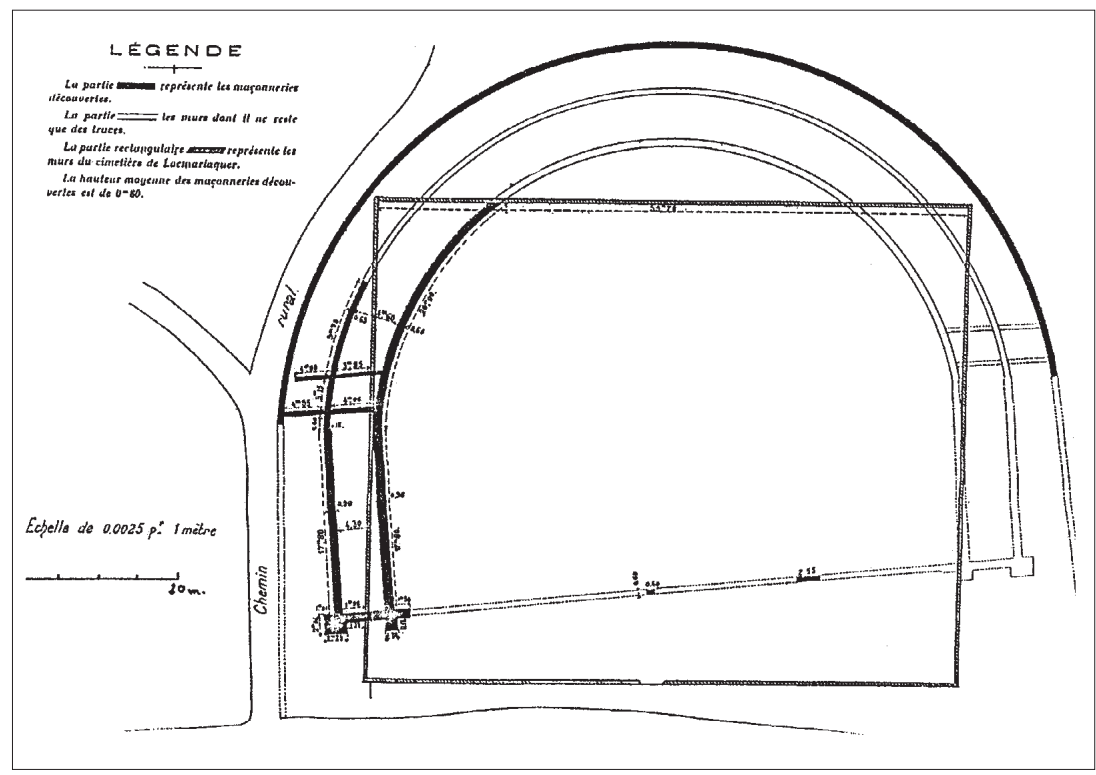

Le dégagement partiel de la partie sud du théâtre a également permis à Gustave Thomas de Closmadeuc de recueillir des indices matériels (notamment plusieurs urnes en terre et en verre contenant des ossements calcinés) qui semblent indiquer une réutilisation d'une partie de l'édifice au Bas Empire comme lieu de nécropole :

"M. Mahé qui a assisté à la découverte de tous les objets, nous a dit et vous répétera que les vases, qui renfermaient des cendres et des ossements, ont été recueillis le long et dans les encoignures des murs romains. Ils n'étaient posés tout droit non pas sur le roc naturel, mais à une certaine hauteur au-dessus, au milieu des décombres. La petite urne en verre qui contenait des ossements humains incinérés a été trouvée en dehors du monument, dans l'angle extérieur formé par le mur de l'hémicycle et celui de la façade. Il n'est pas jusqu'aux fragments de briques qui recouvraient et entouraient les vases funéraires qui ne semblent avoir été empruntés aux débris du monument et

18. Closmadeuc, Gustave Thomas de, "Théâtre romain de Locmariaquer ", Bulletin de la Société polymathique du Morbihan, 1893, p. 184-186. 
choisis tels quels pour protéger le contenu. Dès lors, on est porté à penser que ces enterrements de quelques vases cinéraires ont dû être effectués à une époque postérieure à la construction du théâtre et qu'on a précisément préféré les encoignures du monument déjà en ruine comme point de repère ${ }^{19}$."

Le plan de l'édifice partiellement dégagé par G. de Closmadeuc montre que la cavea se prolonge de manière rectiligne de part et d'autre de l'orchestra débordant largement le cadre de l'hémicycle. Cette caractéristique laisse supposer que la scène devait être située sensiblement à l'emplacement de l'actuelle route d'Auray (D 781) c'est-à-dire à une distance relativement importante de la cavea. Cette particularité pourrait alors indiquer que ce monument serait plus apparenté à une sorte d'amphithéâtre dont la scène nécessite un plus grand espace que celui d'un théâtre.

\section{L'édifice du Er Hastel}

À la sortie du bourg, le long de la route de Kerpenhir, on peut apercevoir aujourd'hui encore dissimulés dans des friches les restes de l'édifice de Er Hastel dont une partie était encore en élévation au début du XIX ${ }^{\mathrm{e}}$ siècle. Les ruines du "Castel César " constituent un ensemble de vestiges figurant en A, B, C sur le plan dressé en 1727 par M. de Robien. À cette époque, il semble que certaines parties aient été encore apparentes mais dans un état de délabrement considérable.

Malgré cela, les portions conservées en élévation permettaient encore de distinguer des éléments architecturaux caractéristiques qui semblaient correspondre à des fourneaux et étuves ( $\mathrm{P}$ et $\mathrm{Q}$ du plan) ce qui permit à M. de Robien de penser qu'il pouvait s'agir des restes d'un édifice de bain :

"Des deux montagnes, qui sont l'une au nord, l'autre au sud, la dernière conserve la dénomination de butte de César. Les ruines qui n'en sont pas loin et dont on joint ici le dessin C B portent encore le nom de Castel-César (on l'appelle aussi château de Kaër), ce dernier ayant été anciennement bâti sur les ruines de cette grande et ancienne ville dont les restes se trouvent presque partout confondus. Mais ces ruines (du Castel-César) qui sont les plus considérables de ce qui reste aujourd'hui sont tellement détruites et défigurées, qu'on ne saurait en reconnaître le véritable usage. On pourrait seulement croire qu'il y avait là des bains dont la partie voûtée servait d'étuve. Tout ce qu'on peut dire de plus assuré c'est que ces bâtiments étaient très étendus. Il y a bien de l'apparence qu'ils communiquaient d'un côté à ceux dont on voit des restes de murs vers la chapelle Saint-Michel ${ }^{20} \ldots$..

En 1987, des sondages ont permis d'examiner le mur de la façade méridionale de l'édifice et celui de sa partie orientale. Il est apparu clairement que la partie basse était construite en petits moellons de granit et arases de briques (opus mixtum) qui ne laissent aucun doute sur l'origine antique

19. Ibidem, p. 190-191.

20. Closmadeuc, Gustave Thomas de, "Le Président de Robien... », art. cit., p. 51-52. 
de ces maçonneries. Ces dernières supportaient une élévation dont le mode architectural différent semblait correspondre à une utilisation tardive du bâtiment. Les vestiges identifiables de ce bâtiment sont très incomplets. Ils constituent un ensemble linéaire de forme rectangulaire orienté nordest/sud-ouest, d'environ 70 mètres de long pour une largeur moyenne de 12 mètres. La connaissance imparfaite de cet édifice ne permet pas d'en préciser sa véritable destination mais son aspect relativement imposant pourrait peut-être indiquer un usage public.

En réalité les vestiges qui émergent sur le site du Lehuie, sont très vraisemblablement postérieurs à l'époque gallo-romaine et leur persistance jusqu'au début du XIX ${ }^{\mathrm{e}}$ siècle accrédite la thèse de l'occupation secondaire du site au Moyen Âge et jusqu'à la fin du XvI ${ }^{\mathrm{e}}$ siècle ${ }^{21}$. On situe traditionnellement l'emplacement du château (ou manoir) de la baronnie des Kaër ${ }^{22}$ dans cette partie du bourg.

\section{Les vestiges de la maison Le Rol}

C'est dans le centre du bourg qu'en 1853 des travaux de terrassement réalisés dans un jardin permirent de mettre au jour très partiellement, les restes d'un édifice thermal gallo-romain (maison Le Rol). Cette découverte fortuite fut immédiatement signalée par le Maire de la commune au Préfet du Morbihan qui s'adressa au Président de la Société Polymathique du Morbihan afin "qu'une commission ou au moins un membre de cette Société fut envoyé sur les lieux ${ }^{23}$ ". Cette dernière dépêcha sur place son secrétaire le $\mathrm{D}^{\mathrm{r}}$ Fouquet qui exécutera deux plans des vestiges complétés d'un commentaire particulièrement détaillé de ces observations (fig. 6).

21. Le 17 janvier 1598 une enquête de la cour d'Auray à la requête de René de Malestroit, sire et baron de Kaër, vicomte de Kerambourg et de Pluvigner, constate que les châteaux de Kaër et de Kerambourg ont été pillés et ruinés par les sieurs d'Arradon, de Camors, d'Asserac et leurs Vassaux (Réformation du domaine de 1684, arrêt du 8 mars 1763, du Grand Conseil entre le duc de Penthièvre et M. de Robien).

22. Les biens de cette ancienne et puissante maison passèrent vers le milieu du XIV ${ }^{\mathrm{e}}$ siècle, dans une branche de celle de Malestroit, qui les transmi par une héritière à celle de Montalais. La baronnie de Kaër et la vicomté de Kerambourg, qui y étaient annexées, furent acquises en 1727 par M. Christophe-Paul de Robien (Ogée, 1843).

23. «En défonçant le sol d'une cour particulière pour en faire un jardin, Louis Monnier, employé des douanes, payé par M. Le Rol, capitaine marin, à qui appartient le terrain, mit à nu d'abord des murs qui ne ressemblent en rien à ceux de nos jours, ensuite des tuiles de formes extraordinaires, puis des ciments d'une composition insolite, enfin des dallages en marbre et un conduit souterrain. M. le Préfet du Morbihan, prévenu de cette découverte par M. le Maire de Locmariaquer, et désirant être édifié sur son importance, s'adresse par lettre à M. le Président de la Société polymathique, afin qu'une commission, ou du moins un membre de cette société fût envoyé sur les lieux. Le 25 janvier et le 16 mai 1853, le $\mathrm{D}^{\mathrm{r}}$ Fouquet s'est transporté à Locmariaquer et a levé deux plans des découvertes opérées successivement... ", Des monuments celtiques et des ruines romaines dans le Morbihan, Arch. dép. d'Ille-et-Vilaine, T 184. 
Figure 6 - Plan des vestiges de la maison « Le Rol " levé le 25 janvier et le 16 mai 1853 par le $D^{r}$ Fouquet de la Société Polymathique du Morbihan

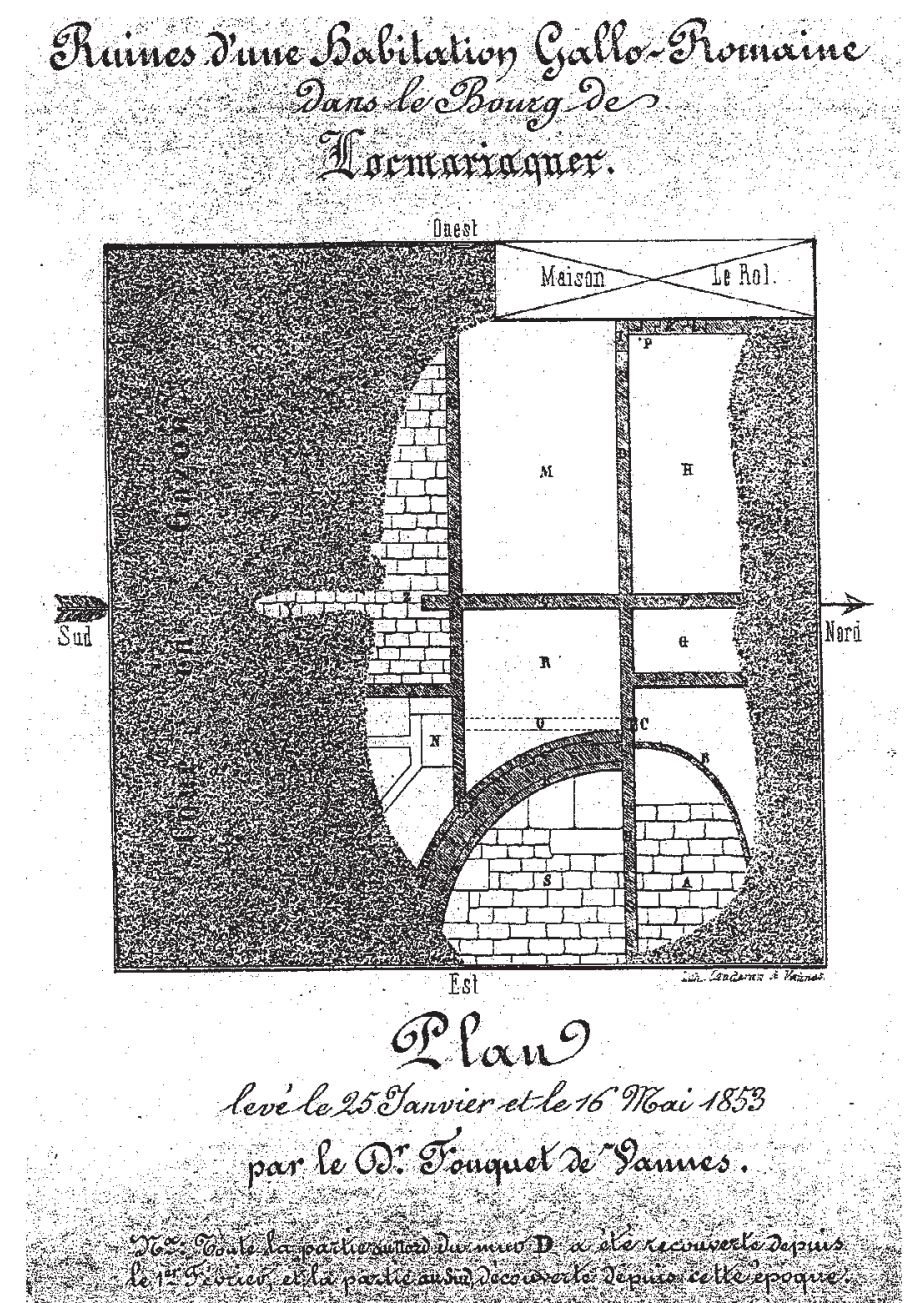

La partie de bâtiment dégagée était dans un état de conservation exceptionnel. Toutes les pièces étaient encore pavées de petites dalles de calcaire fin et les murs garnis de stucs peints ou de dalles d'ardoises parfaitement ajustées les unes aux autres. L'une des pièces la mieux conservée, était une sorte d'absidiole de deux mètres de profondeur au fond de laquelle se trouvait une petite voûte " toute noircie de fumée " qui formait un conduit à travers le mur. L'ensemble des vestiges était orienté est-ouest. La plupart des pièces qui formaient l'édifice furent sondées afin de connaître l'épaisseur des maçonneries et leur mode de construction. 
" [...] Les plus belles pièces en $\mathrm{H}$, en M. et en $\mathrm{Z}$ étaient sans aucun doute destinées aux maîtres; car c'est dans ces pièces qu'on remarquait les carrelages les plus soignés et les lambris les mieux décorés, autant qu'on a pu juger par les enduits adhérents aux parties inférieures des murs au-dessus des marbres; ces enduits d'une épaisseur de $3 \mathrm{~cm}$, recouverts d'une mince couche de stuc, étaient peints à fresque et présentaient des couleurs variées, disposées par bandes alternativement larges et étroites. On observait des peintures de différentes couleurs, du moins, on a trouvé des parois portant des bandes violettes, d'autres des bandes vertes et enfin d'autres encore ornées de bandes jaunes, toutes sur stuc. Ces peintures étaient aussi bien conservées et aussi fraîches qu'elles pourraient l'être dans une ruine toute récente. Quant aux pièces $\mathrm{S}$ et $\mathrm{N}$, elles avaient évidemment des destinations spéciales que nous allons essayer de découvrir, quoiqu'elles ne soient pas entièrement déblayées. À quel usage pouvait être consacrée la pièce S, située, construite et carrelée tout autrement que les autres, et dont les murs formant les parois étaient revêtus de grandes dalles en schiste, dans leur partie inférieure?

Si nous prenons en considération la couleur fuligineuse des pierres inférieures du mur circulaire qui la touche, la petite voûte toute noircie de fumée qu'on observait à sa base et enfin une ouverture carrée qui formait un conduit à travers ce mur, et se dirigeait vers l'ouest, on est porté à penser que là, devait être l'hypocauste dont la chaleur allait se répandre partout $[\ldots]^{24}$."

Au sud-ouest de l'église, une anomalie topographique individualise fortement un îlot limité à l'est par la rue Lafayette. Cette singularité pourrait peut-être traduire l'indice de l'existence d'un édifice de grande taille à cet emplacement. Il n'est d'ailleurs pas exclu que les vestiges de " la maison Le Rol " puissent appartenir à un plus vaste ensemble dont la surface dépasse largement le strict cadre de la zone explorée. On note également dans ce périmètre, l'existence de plusieurs " tourelles " alignées un peu en retrait du littoral ${ }^{25}$. Sur le plan levé en 1727 par M. de Robien la présence de l'une d'entre elles figure le long de l'actuelle rue Lafayette (K du plan de Robien). Cette dernière d'un diamètre de 12 pieds était associée à des murs dont le tracé était parallèle à cette rue. L'ensemble de ces éléments permet de penser qu'il existe dans ce secteur un édifice dont l'architecture monumentale a subsisté assez longtemps pour marquer de son empreinte l'assiette topographique et la forme modulaire du parcellaire dans le centre bourg ${ }^{26}$.

24. FOUQUET $\mathrm{D}^{\mathrm{r}}$, Des monuments celtiques..., op. cit.

25. M. de Robien signale l'existence d'une seconde tourelle au sud-est du bourg à la limite de l'ancien rivage et de l'estran. Elle est accolée en flanquement dans l'angle sudest d'un petit édifice de forme quadrangulaire qui semble isolé et légèrement en retrait par rapport à l'agglomération ( $\mathrm{N}$ et $\mathrm{M}$ du plan de Robien) (fig. 2).

26. L'étude architecturale de l'église de Locmariaquer pourrait peut-être permettre de définir si l'abside du chœur dont le mode de construction en petit appareil (opus uitattum) contraste fortement avec le reste de l'édifice, est antérieure à la partie $\mathrm{xl}^{\mathrm{e}}$ siècle (croisée des transepts). En effet, il n'est pas impossible que cette partie de l'église puisse correspondre aux restes d'un édifice plus ancien contre lequel elle se serait en quelque sorte "greffée ", ce qui pourrait expliquer l'absence d'orientation symbolique de l'église. 


\section{Un probable fanum}

En 1885, G. de Closmadeuc intrigué par la présence d'un mur antique muni de cinq contreforts signalé par M. de Robien (I du plan de Robien) dans un terrain nommé Parc-er-Belek proposa au propriétaire des lieux de l'autoriser à pratiquer une fouille. Ce dernier refusa d'emblée cette requête malgré les compensations généreuses qui lui étaient offertes en échange de cette exploration. Malgré tout, le propriétaire entama lui-même une fouille dans son propre terrain. Après un certain temps, il mit au jour le mur signalé par M. de Robien qui s'avéra être effectivement garni de plusieurs contreforts sur son parement externe (nord). Les remblais qui s'appuyaient contre ce mur formaient une masse considérable de matériaux de construction parmi lesquels figuraient « des quantités considérables de débris de poteries gallo-romaines, d'ossements d'animaux et de bois de daim ". Ce même remblai livra un bloc de calcaire d'une cinquantaine de centimètres de hauteur qui s'avéra correspondre à la partie inférieure d'un autel sur laquelle figuraient gravées les lettres V. S. L. M. (Votum. Solvit. Libenter. Merito). Il fut également découvert un fût de colonne en calcaire garni de sa base. Cette pièce lapidaire était similaire à une autre découverte précédemment dans ce même terrain.

C'est apparemment la mise au jour du fragment d'autel présentant sur l'une de ses faces une dédicace épigraphique et celles de colonnes qui suscita l'idée du fanum dans ce secteur du bourg. Dans l'hypothèse de l'existence de ce monument, il serait assez tentant de voir dans le mur relevé par M. de Robien et signalé par d'autres auteurs après lui, un élément du péribole. Ce dernier ferait également office de mur terrasse destiné à retenir les terres situées en surplomb par rapport aux terrains environnants.

Certains monuments mégalithiques situés dans le centre bourg (et de ce fait intégrés dans l'espace urbain) ont également livré du mobilier galloromain parfois en abondance. Le dolmen du Mané Rutual qui est situé à une distance de 150 mètres au nord du Parc-er-Belek constitue l'un des plus imposants monuments de Locmariaquer. Sa galerie qui est constituée de 36 supports se développe sur une longueur totale de 23 mètres. Elle est recouverte de six dalles dont une de taille colossale d'une surface de près de $45 \mathrm{~m}^{2}$. Ce dolmen fut exploré à plusieurs reprises notamment par Gustave Thomas de Closmadeuc en 1885. Le mobilier recueilli dans la couche supérieure du comblement interne était essentiellement composé de tégulae et de céramiques gallo-romaines. Figurait également parmi ce matériel, un très grand nombre de figurines en terre blanche, vénus anadyomène et latone, brisées. La présence en quantité d'un tel type de mobilier pose nécessairement la question sur la fonction cultuelle du monument à l'époque antique. En effet, il n'est pas impossible qu'il puisse s'agir là de l'expression de pratiques religieuses indigènes romanisées. 
Figure 7 - Plan de l'édifice gallo-romain et des vestiges gaulois mis au jour à l'emplacement de l'école du Votten

(plan et dessin : L Pirault, 1996)

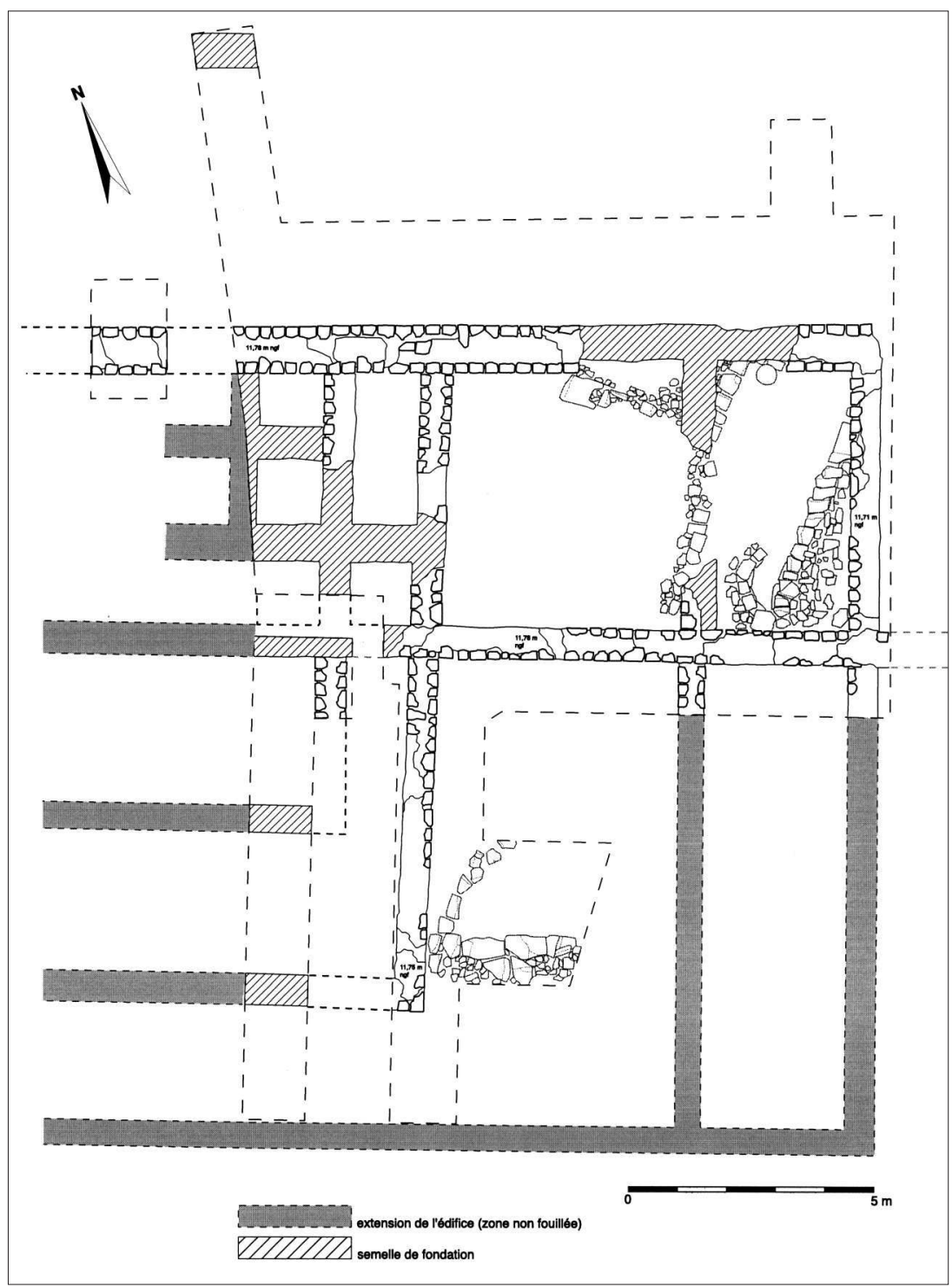

\section{Les fouilles du Votten}

C'est M. de Robien qui en 1727 indique la présence d'un bâtiment antique sensiblement à l'emplacement de l'ancienne école publique du Votten (D et E du plan de Robien). Selon cet auteur, ces substructions pou- 
vaient constituer le prolongement occidental de l'édifice du Er Hastel (A, $\mathrm{B}, \mathrm{C}$ du plan de Robien) tout comme les vestiges antiques découverts sous la chapelle Saint-Michel (G du plan de Robien) ${ }^{27}$.

En 1996, une fouille de sauvetage réalisée à l'emplacement de l'ancienne école publique située dans la partie sud-ouest du centre bourg a permis de mettre au jour sur une surface de $320 \mathrm{~m}^{2}$ les restes très arasés d'un bâtiment gallo-romain (fig. 7). Le mauvais état de conservation de ces vestiges résulte de l'aplanissement du terrain avant la construction de l'école et de ses annexes. On peut donc présumer qu'antérieurement, ces vestiges antiques devaient présenter un état de conservation comparable à celui observé par M. de Robien au XVIII ${ }^{\mathrm{e}}$ siècle. Si l'on se rapporte aux observations faites par M. de Robien, il semblerait que les sols de la partie ouest du bâtiment aient été encore en place à cette époque puisqu'il en fait une description assez précise "salon pavé des grandes briques marquées $\mathrm{V}$ sur un fond de ciment d'une dureté extrême ".

Le plan fragmentaire n'a pas permis de connaître la fonction exacte du bâtiment. Toutefois, plusieurs éléments semblent indiquer qu'il pourrait s'agir d'une partie d'un édifice appartenant à une plus vaste infrastructure comme l'indique le prolongement de certains murs dans les parcelles voisines. D'autre part, la distribution de certaines pièces évoque celle d'un ensemble composé de petites boutiques de forme rectangulaire, alignées les unes à côté des autres. On remarque également concernant l'une d'entre elle (la plus grande) la présence d'un aménagement constitué par un caillebotis qui pourrait correspondre à un système de vide sanitaire. La fouille a révélé l'existence d'au moins deux phases de construction qui semblent s'être succédé sur une courte durée. L'absence de tout mobilier archéologique associé à chacun de ces épisodes n'a pas permis d'en établir la chronologie avec précision.

S'il est difficile d'interpréter le plan de cet édifice, il reste néanmoins la possibilité de confronter les données cartographiques anciennes et scientifiques récentes tout en tenant compte de l'incertitude des premières et de la réalité des secondes. Il résulte une superposition intéressante des vestiges qui permet de valider la fiabilité des données cartographiques anciennes en notre possession.

\section{L'occupation gauloise du site}

Contrairement à l'édifice gallo-romain qui n'a livré aucun élément matériel permettant d'établir sa chronologie historique, la fouille des niveaux antérieurs nous a permis de recueillir un abondant mobilier céramique

27. M. de Robien acheta l'ancienne chapelle Saint-Michel à la famille de Kaër. En 1840, lors de travaux de réfection on découvrit un pan de mur gallo-romain composé d'assises régulières de moellons alternant avec des briques. Directement au sud de la chapelle, il fut également mis au jour plusieurs murs antiques ainsi que les restes de deux fours de forme pyramidale. 
attribuable à la fin de l'âge du Fer. Cette occupation qui semble se limiter à la partie nord de la parcelle forme un ensemble de vestiges difficiles à commenter du fait de leur représentation imparfaite. Il s'agit des restes d'un aménagement composé par deux alignements parallèles formés de blocs de granit grossièrement équarris. L'une de ces deux structures constitue un parement rectiligne qui maintient un épais blocage rocailleux. Ces vestiges ne semblent pas correspondre directement à de l'habitat tel qu'on peut le concevoir pour ces périodes (fond de cabane sur solin en pierre, architecture de terre et de bois sur poteaux plantés). La topographie pentue du terrain qui présente une forte déclivité vers le sud suggère plutôt qu'il s'agit d'aménagements étagés qui pouvaient supporter des habitations ${ }^{28}$. Les murs environnants constitueraient alors des infrastructures annexes limitant jardins ou enclos autour de ces plates-formes.

Le mobilier recueilli dans ce niveau d'occupation est essentiellement composé de céramiques communes non tournées ${ }^{29}$ qu'accompagnent de nombreuses amphores italiques (de type Dressel 1). Il est intéressant de noter la présence d'importation tarraconaise précoce (Pascual I) et d'un exemplaire de col d'amphore de Lipari (101.2) portant la marque probable du potier PORTINENTI (APOL) et datable des dernières décennies du premier siècle avant notre ère (15-10 av. J.-C.) (fig. 8).

Depuis la fin du XIX ${ }^{\mathrm{e}}$ siècle, l'intérêt des érudits s'est essentiellement concentré sur les monuments préhistoriques qui parsèment la commune, délaissant quelque peu les vestiges de l'occupation gallo-romaine. Il en résulte une connaissance approximative d'où émergent seulement quelques édifices que l'on présume publics et qui permettent toutefois d'estimer l'importance de l'occupation antique. En effet, si nos connaissances concernant l'agglomération gallo-romaine de Locmariaquer sont très lacunaires, il n'en reste pas moins que son étendue (évaluée à une vingtaine d'hectares) peut être appréciée à partir de la répartition géographique des seules données recueillies sur le terrain. Si la forme de l'organisation urbaine reste encore ignorée, l'existence d'un parcellaire offrant une dominante identique à celle des vestiges connus pourrait éventuellement indiquer la trace des rues urbaines.

28. Des constructions similaires furent partiellement dégagées dans les années 19861987 au cours d'une fouille réalisée sur le site de Pluguffan (Finistère). Elle permit de mettre au jour des aménagements en terrasse datables de la transition Tène finale/galloromain précoce, construits sur le flanc d'un coteau peu élevé. MENEz, Yves, "Sondage sur un habitat datable de la transition la Tène finale-gallo-romain précoce ", F. AudouzE et Olivier Buchsennschutz (dir.), Architectures des âges des métaux, fouilles récentes, Dossier de protohistoire $\mathrm{n}^{\circ}$ 2, Paris, 1988, p. 111-120.

29. Parmi ce mobilier figurent quelques céramiques fines. Il s'agit de vases tournés dont la surface extérieure est ornée soit par des cordons en relief ou par des croisillons tracés à la baguette. Certains autres exemplaires présentent des enductions au graphite sur le haut de la panse. 
¿ ช.

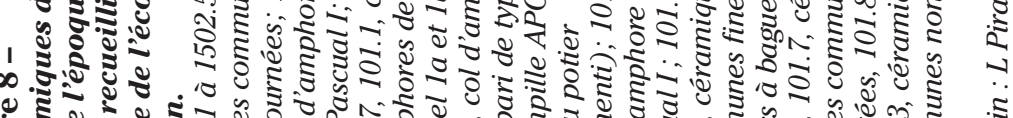

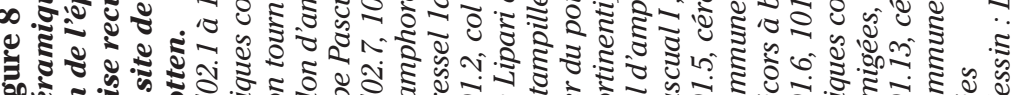

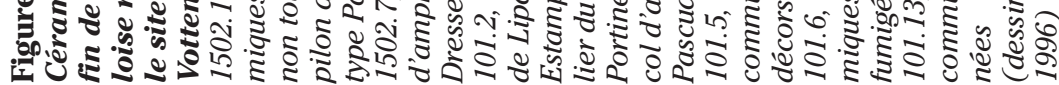

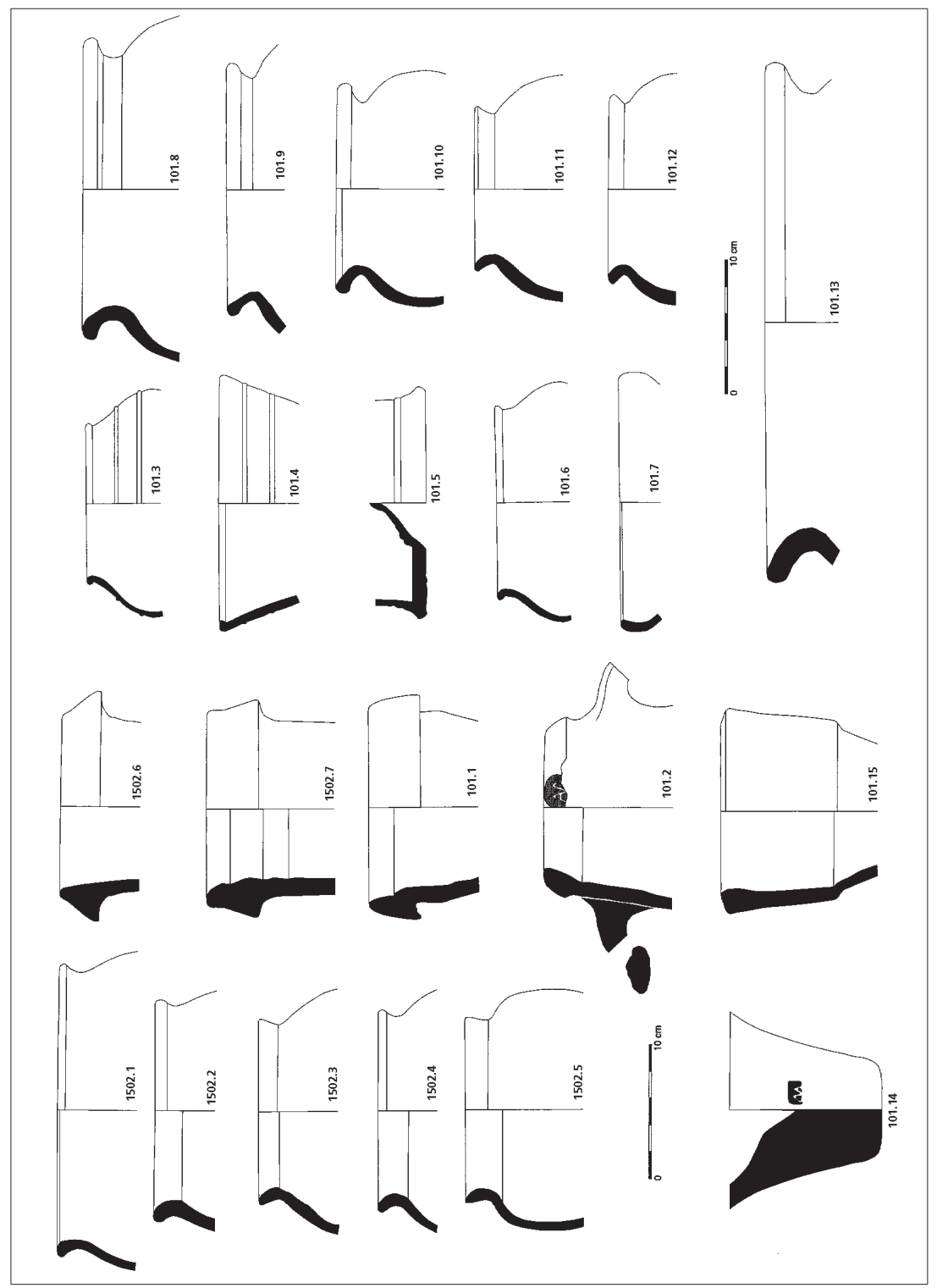


Il est important de remarquer que la ville antique est implantée sur un lieu de forte concentration de monuments mégalithiques; au nord-ouest, figurent le Mané-Lud, la Table des Marchand, le Grand Menhir et le tumulus d'Er-Grah; le dolmen du Mané-Rutual est situé dans la zone urbaine gallo-romaine présumée ainsi que le menhir du Men-Bronso; au sud-est, à la sortie du bourg se dresse le Mané Er-H'roueg dont le sommet constitue l'un des points culminants de la commune. Le choix de l'implantation de l'agglomération au sein d'un aussi important regroupement de monuments n'est peut-être pas le fait d'un simple hasard. Il peut s'agir là d'un point primordial qui pourrait traduire la fonction première de cette petite ville (sanctuaire indigène?). Toutefois, il est indéniable que la géographie se révèle d'une importance fondamentale. L'agglomération est construite dans une anse protégée des vents dominants d'ouest ce qui constitue un atout important pour un port d'embarquement et un abri privilégié pour les embarcations. On peut en effet penser qu'elle devait participer au réseau commercial de communication et de distribution des ports de la façade atlantique. Sa situation à l'entrée du golfe du Morbihan est également déterminante puisqu'elle constitue un point d'observation privilégié qui permettait de contrôler l'accès de la navigation vers le chef lieu de cité Darioritum (Vannes). 


\section{RESUME}

Locmarinquer (56) se situe à l'extrémité sud-est d'une péninsule qui ferme à l'ouest le seul accès possible par la mer du golfe du Morbihan. Cette commune qui est située à peu de distance de Vannes (Darioritum) au seuil du golfe du Morbihan, fait partie intégrante de la Cité des Vénètes.

Elle s'étend de la rivière d'Auray à l'est, à la baie de Quiberon à l'ouest et au sud. Cette situation géographique remarquable lui confère nécessairement un rôle important le long du littoral atlantique.

Cette région a subi de profondes modifications résultant des variations climatiques aux conséquences multiples. Lors des transgressions et régressions marines, les lignes de rivage ont connu des fluctuations importantes qui ont fortement influencé l'implantation des premières populations de cette partie de la Bretagne.

\section{ABSTRACT}

Locmarinquer (56) is situated on the south east end of a peninsule which closes westward the only access possible by the sea to the bay of Morbihan. Near Vannes (Darioretum) this commune belongs to the "Cité des Vénètes".

It spreads from the river of Auray eastward to the bay of Quiberon westward and southward. Owing to this remarkable geographic situation, it plays an important part all along the atlantic littoral. This region was deeply altered due to climatic variations with multiple effects. Because of the ebb and flow, the coastline has considerably fluctuated. It strongly influenced the settling of the first populations in this part of brittany. 
